\title{
Rainfall as proxy for evapotranspiration predictions
}

\author{
Bruno Collischonn ${ }^{1}$ and Walter Collischonn ${ }^{2}$ \\ ${ }^{1}$ Agencia Nacional de Aguas, Brasilia, 70610-200, Brazil \\ ${ }^{2}$ Instituto de Pesquisas Hidraulicas, Porto Alegre, 91501-970, Brazil
}

Correspondence to: Bruno Collischonn (bruno.collischonn@ana.gov.br)

Published: 17 October 2016

\begin{abstract}
In this work, we evaluated the relationship between evapotranspiration and precipitation, based on the data recently made available by the Brazilian Meteorological Institute. ETP tend to be lower in rainy periods and vice-versa. This relationship was assessed both in physical and statistical ways, identifying the contribution of each explaining variable of ETP. We derived regression equations between monthly rainfall and ETP, which can be useful in studies where ETP time series are not available, such as reservoir design, irrigation management and flow forecast.
\end{abstract}

\section{Introduction}

Evaporation and evapotranspiration (E/ETP) are components of the water cycle, corresponding to the transfer of water from the terrestrial surface to the atmosphere. Quantitative information about these components is used in several problems involving water resources, such as crop management (both irrigated and non-irrigated), reservoir design and operation, flow forecast and so on. However, E/ETP data availability is sparse both in time and space, as well as the data needed to estimate it (solar radiation, wind speed, relative humidity, air temperature and atmospheric pressure) using consecrated methods like Penman-Monteith (Shuttleworth, 2012).

Moreover, E/ETP shows a lower variability, compared with precipitation. These factors (data scarcity and low variability) lead to the fact that many studies in water resources in Brazil use simple evaporation vectors, i.e. long-term monthly averages of E/ETP, instead of time series. When evaluating reservoir yields, for example, most studies in Brazil use $E / E T P$ averages. It is straightforward to conclude, however, that this approach leads to an underestimation of reservoir yields, since during a sequence of dry years, which normally defines the magnitude of reservoir yields, real $E / E T P$ is greater than the average. On the other hand, in wet years, when $E / E T P$ is lower than average, one can expect frequent reservoir spilling; thus, excess volumes do not compensate interanually.
The Brazilian hydropower network, which accounts for more than $70 \%$ of power supply in the country, is designed to minimize the risk of power shortages in the case of repetition of a so-called critical hydrological period, which was a very dry sequence of years in the country as a whole, from 1949 to 1956 . But hydrological data during that period was very scarce, leading to the need of hydrological modelling, mostly using $E /$ ETP averages. Better estimates of $E / E T P$ would allow a more real assessment of the supply capacity, as well as a better estimation of historical inflows.

Again, given the low variability of $E / E T P$, it is often assumed that the errors incurred by using averages are lower than the estimation of E/ETP itself (as well as the estimation of other hydrological variables), making this approach widely accepted.

However small, interannual variability in E/ETP does exist, and it is in some ways linked to the variability of precipitation. In general, there is an inverse relationship of rainfall and E/ETP in a given time interval. As a consequence, the rainfall-runoff transformation process becomes nonlinear, as stated by Tucci (1998), since an increase of, say, $100 \mathrm{~mm}$ in monthly precipitation leads to an increase in runoff higher than $100 \mathrm{~mm}$, because of the decrease of $E / E T P$ (besides other non-linearities). The inverse relationship between these two variables has not been very much investigated in the literature, one exception being the work by Mudiare (1985).

Therefore, a way of overcoming E/ETP data scarcity, particularly in developing regions, is to establish statistical re- 
lations between $E / E T P$ and rainfall. Once this relationship exists, E/ETP time series are obtained by applying the regression equation with the precipitation time series as input.

Recently, the Brazilian National Institute of Meteorology (INMET) has put its daily hydrometeorological time series available for research purposes. Data since 1961 over 290 stations around the country can be obtained through the internet after registering, attending a long-time demand of water managers and research community in Brazil.

In the present work, some statistics were calculated using this database, in order to identify a more physical explanation for the inverse relationship between rainfall and $E / E T P$, by quantifying the magnitude of the underlying meteorological variables both in dry and wet days.

Moreover, regression equations between monthly E/ETP and rainfall at each station were derived. The goal is to obtain a better comprehension of the relationship, as well as generating an E/ETP database for the refinement of water resources studies, as an alternative of the use of averaged data.

\section{Rainfall and E/ETP}

The Penman-Monteith equation, which is considered to be the most suited method for quantifying E/ETP (Smith, 1991), can be rewritten as a function of five meteorological variables:

$$
E / \mathrm{ETP}=f\left(T_{\mathrm{med}}, \mathrm{UR}, \mathrm{PA}, \mathrm{VV}, \mathrm{RAD}\right)
$$

where $T_{\text {med }}$ is the mean air temperature, UR is the relative humidity, PA is the atmospheric pressure, $\mathrm{VV}$ is the mean wind speed, and RAD is the incident solar radiation. In our work, incident radiation is calculated from sunshine duration, in hours.

The inverse relationship between rainfall and E/ETP can be explained physically, through the variation of its explaining variables. First, rainy days are necessarily cloudy, reducing the incoming radiation. This is the main factor explaining the inverse relationship, since solar radiation (or sunshine duration) is the main driver of the process of evaporation and evapotranspiration (Shuttleworth, 2012).

Rainy days also generally show higher relative humidity. This leads to a decrease of $E / E T P$, since higher humidity causes an increase in vapour pressure (neighbouring air moisture is closer to saturation).

The effects of the remaining variables are not so evident. Mean temperature, for example, is positively correlated with $E / E T P$, since increases vapour saturation pressure (the amount of moisture that can be stored in the surrounding air). However, FAO's recommendation is to use the average between the minimum and maximum daily temperature as an estimate of mean temperature. In rainy days, lower radiation tend to decrease maximum temperatures, but during the night, outgoing longwave radiation is retained in the atmosphere by the clouds, actually increasing minimum temperatures (Allen et al., 1998), at least in temperate climates. Thus, the relevance of mean air temperature in the rainfall- $E$ /ETP relationship is relative.

In the case of the other variables (atmospheric pressure and wind velocity), it is harder to establish a correlation intuitively, as made above. The magnitude of each meteorological variable in dry and wet years were statistically tested as follows.

\section{Statistical analysis of ETP's explaining variables}

For each station, daily time series were separated between dry and rainy days, the latter defined as days with precipitation lower or equal than $5 \mathrm{~mm}$. For each of the five variables, we tested the hypothesis that the mean of the variable in dry days is equal to the mean in rainy days (null hypothesis), using Student's $t$ test of the means implemented in Matlab ${ }^{\circledR}$ for a $5 \%$ significance level. If the null hypothesis was rejected, it was verified that the mean in rainy days was higher or lower than in dry days.

Seasonality was also accounted for, by performing the tests for each month of the year. Months where less than $5 \%$ of the days were rainy were discarded (typically the period between July and September in the Southern Hemisphere), in order to avoid non-rejection of the null hypothesis only because of the small size of samples.

INMET stations were grouped according to the five Brazilian climate zones (IBGE, 2013). For each zone, we calculated the frequency of months/stations where magnitude was higher in dry and rainy days, as well as the percentage of months were the null hypothesis could not be rejected (in this case, we considered that no significant difference exists).

\section{Results - statistical correspondence}

\section{Wind speed (or: "do rainy days have stronger wind?")}

Table 1 shows the condensed results of the hypothesis test for wind velocity. For each climate zone, we calculated the frequency of months/stations where wind was stronger during rainy and dry days, as well as no significant difference.

In most regions, there is no difference between wind velocity during rainy and dry days. In the semi-arid and coastal northeast, stronger wind occurs slightly more frequently during dry days. In the more temperate south, it is the other way round. In general, it was observed that coastal stations showed stronger wind during dry weather, while stations in hillier regions have stronger winds more frequently during rainy days.

Wind velocity influences $E$ /ETP by removing moisture from the surface, bringing less saturated air. Thus, higher velocities result in higher $E / E T P$. As seen, dry days, more often than not, show stronger wind velocity, which also helps to explain the negative relationship between rainfall and E/ETP. 
Table 1. Frequency of stronger wind during dry and rainy days.

\begin{tabular}{lrrr}
\hline Zone & Dry & No diff. & rainy \\
\hline Temperate & $15 \%$ & $40 \%$ & $46 \%$ \\
Tropical & $27 \%$ & $59 \%$ & $14 \%$ \\
Coastal NE & $41 \%$ & $49 \%$ & $10 \%$ \\
Semiarid & $55 \%$ & $38 \%$ & $7 \%$ \\
Equatorial & $32 \%$ & $56 \%$ & $12 \%$ \\
Brazil & $34 \%$ & $50 \%$ & $15 \%$ \\
\hline
\end{tabular}

Table 2. Frequency of higher maximum temperature during dry and rainy days.

\begin{tabular}{lrrr}
\hline Zone & Dry & No diff. & rainy \\
\hline Temperate & $98 \%$ & $2 \%$ & $0 \%$ \\
Tropical & $95 \%$ & $5 \%$ & $0 \%$ \\
Coastal NE & $80 \%$ & $20 \%$ & $0 \%$ \\
Semiarid & $79 \%$ & $20 \%$ & $1 \%$ \\
Equatorial & $60 \%$ & $38 \%$ & $2 \%$ \\
Brazil & $85 \%$ & $15 \%$ & $1 \%$ \\
\hline
\end{tabular}

Temperature (or: "are dry days warmer?")

Statistical analyses were performed considering minimum, maximum and mean daily temperature. Table 2 shows the analysis for the maximum temperature. In almost $90 \%$ of the cases, maximum temperature was significantly higher during dry days than during rainy days, more so in the temperate south and less so in the equatorial amazon. It confirms the intuitive notion, also stated by Allen (1998), that dry days have maximum temperatures higher than rainy days.

For minimum temperatures, aggregate results are shown in Table 3. Its results show that the assumption by Allen (1998), that rainy/cloudy days have higher minimum temperatures, is valid for temperate climates, but not necessarily for tropical/equatorial regions. Here, very often minimum temperatures were higher in dry days.

In the average, results shown in Table 4 allow the conclusion that dry days tend to be warmer in all regions of the country. As seen, this is in part due to higher maximum temperatures, as expected, but in some parts also due to higher minimum temperatures even in dry days, contrary to some literature, notably Allen et al. (1998).

This also helps to explain the negative relationship between rainfall and E/ETP, since, as seen, temperature is positively correlated with $E / E T P$.

\section{Radiation/Sunshine duration and relative humidity}

In the case of sunshine duration (used here to estimate incoming radiation), the correlation is quite intuitive, and is confirmed by the statistical analysis: in almost $100 \%$ of the months/stations, mean sunshine duration was higher in dry
Table 3. Frequency of higher minimum temperature during dry and rainy days.

\begin{tabular}{lrrr}
\hline Zone & Dry & No diff. & rainy \\
\hline Temperate & $3 \%$ & $18 \%$ & $79 \%$ \\
Tropical & $27 \%$ & $38 \%$ & $36 \%$ \\
Coastal NE & $24 \%$ & $60 \%$ & $16 \%$ \\
Semiarid & $26 \%$ & $60 \%$ & $14 \%$ \\
Equatorial & $43 \%$ & $51 \%$ & $6 \%$ \\
Brazil & $26 \%$ & $44 \%$ & $30 \%$ \\
\hline
\end{tabular}

Table 4. Frequency of higher mean temperature during dry and rainy days.

\begin{tabular}{lrrr}
\hline Zone & Dry & No diff. & rainy \\
\hline Temperate & $61 \%$ & $34 \%$ & $5 \%$ \\
Tropical & $79 \%$ & $18 \%$ & $2 \%$ \\
Coastal NE & $46 \%$ & $53 \%$ & $1 \%$ \\
Semiarid & $46 \%$ & $51 \%$ & $2 \%$ \\
Equatorial & $42 \%$ & $56 \%$ & $2 \%$ \\
Brazil & $61 \%$ & $37 \%$ & $3 \%$ \\
\hline
\end{tabular}

Table 5. Frequency of higher mean sunshine duration during dry and rainy days.

\begin{tabular}{lrrr}
\hline Zone & Dry & No diff. & rainy \\
\hline Temperate & $100 \%$ & $0 \%$ & $0 \%$ \\
Tropical & $100 \%$ & $0 \%$ & $0 \%$ \\
Coastal NE & $100 \%$ & $0 \%$ & $0 \%$ \\
Semiarid & $100 \%$ & $0 \%$ & $0 \%$ \\
Equatorial & $98 \%$ & $2 \%$ & $0 \%$ \\
Brazil & $99 \%$ & $1 \%$ & $0 \%$ \\
\hline
\end{tabular}

days, as seen in Table 5. This is the main factor explaining the inverse relationship between rainfall and E/ETP.

The same happens for relative humidity. Table 6 shows that in $100 \%$ of the cases, relative humidity is higher during rainy days. Since relative humidity is negatively correlated with E/ETP, this also explains the inverse relationship investigated in this paper.

Finally, results for atmospheric pressure are shown in Table 7. For higher latitudes, there is a positive correlation between dry weather and atmospheric pressure, as expected. In regions closer do the Equator; however, this correlation is no longer valid, since there is no statistical difference between pressure during dry and rainy days. The variability of atmospheric pressure also plays a minor role in the variability of ETP; for one side, higher pressure increases the specific weight of air, allowing more moisture to be retained in the surrounding air; on the other hand, it increases the psychrometric constant, which comes into the denominator of the Penman-Monteith equation, thus decreasing ETP. 
Table 6. Frequency of higher relative humidity during dry and rainy days.

\begin{tabular}{lrrr}
\hline Zone & Dry & No diff. & rainy \\
\hline Temperate & $0 \%$ & $0 \%$ & $100 \%$ \\
Tropical & $0 \%$ & $0 \%$ & $100 \%$ \\
Coastal NE & $0 \%$ & $0 \%$ & $100 \%$ \\
Semiarid & $0 \%$ & $0 \%$ & $100 \%$ \\
Equatorial & $0 \%$ & $0 \%$ & $100 \%$ \\
Brazil & $0 \%$ & $0 \%$ & $100 \%$ \\
\hline
\end{tabular}

Table 7. Frequency of higher atmospheric pressure during dry and rainy days.

\begin{tabular}{lrrr}
\hline Zone & Dry & No diff. & rainy \\
\hline Temperate & $88 \%$ & $7 \%$ & $5 \%$ \\
Tropical & $65 \%$ & $27 \%$ & $8 \%$ \\
Coastal NE & $9 \%$ & $60 \%$ & $31 \%$ \\
Semiarid & $18 \%$ & $57 \%$ & $24 \%$ \\
Equatorial & $8 \%$ & $71 \%$ & $21 \%$ \\
Brazil & $44 \%$ & $41 \%$ & $15 \%$ \\
\hline
\end{tabular}

\section{Relations between rainfall and ETP}

Daily time series for ETP were calculated through the Penman-Monteith, based on Shuttleworth (2012) and synthetized by Collischonn et al. (2007), using the meteorological data recently made available by INMET in 290 meteorological stations in Brazil. Incident radiation data was estimated based on sunshine duration. Reference evapotranspiration was calculated adopting an albedo of $0.23 \mathrm{~m}$, surface resistance of $70 \mathrm{~s} \mathrm{~m}^{-1}$ and crop height of $0.12 \mathrm{~m}$ (Allen et al., 1998).

During time intervals where one or more of the variables were missing, ETP was not calculated, the only exception being missing atmospheric pressure. In this case, pressure was estimated based on a seasonal correlation between monthly mean pressure and station elevation. This is considered to be acceptable, given the low influence of this variable over the variability of ETP and the large number of missing data for atmospheric pressure.

ETP data where then accumulated into monthly totals. If missing data accounted for less than 5 days, missing data were filled using the mean ETP of the remaining days. Otherwise, the month was not included in the regression. The same was done for monthly rainfall.

Regression equations were then derived from the paired ETP/rainfall monthly data. Regressions were done for each month of the year, in order to account for seasonality. Given the expected inverse relationship between these variables, the slope of the regression should be negative.

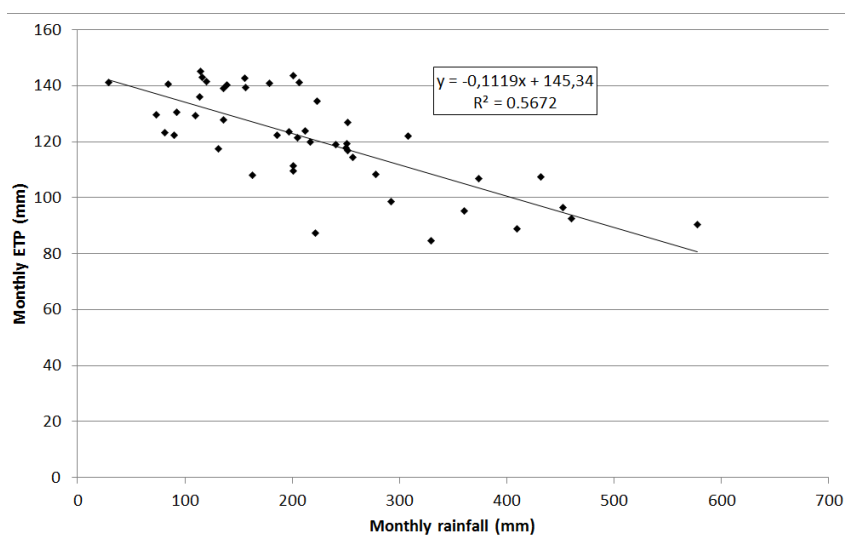

Figure 1. Example of regression for Brasilia, month of January.

Results - relations between rainfall and ETP

Figure 1 shows an example of the resulting regressions, for the meteorological station of Brasilia (tropical Brasil central zone), for the month of January. It can be observed that, as expected, rainfall variability is in general much higher than that of ETP. In this case, while rainfall varied between 29 and $577 \mathrm{~mm} \mathrm{month}^{-1}$, ETP varied between 85 and $145 \mathrm{~mm} \mathrm{month}^{-1}$. The negative slope shown in this example was also obtained for all remaining regressions, emphasizing the inverse relationship between rainfall and ETP.

Although data dispersion is relatively high (also expressed in the low coefficient of determination $R^{2}$, it should be remembered that these regressions are an alternative for the use of long-term averages of ETP for hydrological simulation, i.e. when no time series are available.

This kind of regression is particularly useful during transition months between the dry and rain seasons in tropical regions (typically April/May and September/October in the Southern Hemisphere), which can be either very rainy or very dry, depending on the year. It is also useful in regions where the rain season presents periodical breaks, or periods of 10 to 15 days without rainfall. These periods, usually between December and March, can have very high ETP given the higher extraterrestrial radiation during summer months.

Only during very dry months, like July and August, ETP can have a higher variability, since rainfall is hardly larger than zero. In this case, the regressions are less useful, and the use of ETP averages seem to be more practical.

Figure 2 shows the spatialized values of the $R^{2}$ coefficient for the regression in two months of transition between rainy and dry seasons.

As can be seen, the higher values for the coefficient were obtained in the tropical zone. In the temperate and equatorial zones, the inverse relationship between rainfall and ETP is not so clear, perhaps because of a higher frequency of cloudy, but not necessarily rainy days. 
Table 8. Monthly averages of $R^{2}$ statistics for the regressions in each climate zone.

\begin{tabular}{lrrrrrr}
\hline & Temperate & Tropical-central Brazil & Coastal north east & Semiarid north east & Equatorial & Month average \\
\hline Jan & 0.26 & 0.51 & 0.54 & 0.45 & 0.29 & 0.41 \\
Feb & 0.29 & 0.46 & 0.51 & 0.43 & 0.25 & 0.37 \\
Mar & 0.19 & 0.41 & 0.33 & 0.35 & 0.20 & 0.21 \\
Apr & 0.31 & 0.29 & 0.37 & 0.43 & 0.27 \\
May & 0.18 & 0.16 & 0.41 & 0.45 & 0.20 & 0.29 \\
Jun & 0.08 & 0.19 & 0.34 & 0.27 & 0.18 \\
Jul & 0.17 & 0.15 & 0.38 & 0.17 & 0.18 & 0.19 \\
Aug & 0.11 & 0.15 & 0.32 & 0.14 & 0.13 & 0.15 \\
Sep & 0.26 & 0.28 & 0.30 & 0.23 & 0.11 & 0.23 \\
Oct & 0.28 & 0.33 & 0.43 & 0.21 & 0.18 & 0.26 \\
Nov & 0.31 & 0.33 & 0.30 & 0.25 & 0.27 \\
Dec & 0.27 & 0.31 & 0.38 & 0.31 & 0.21 \\
\hline Zone average & 0.22 & 0.30 & & & 0.28 \\
\hline
\end{tabular}
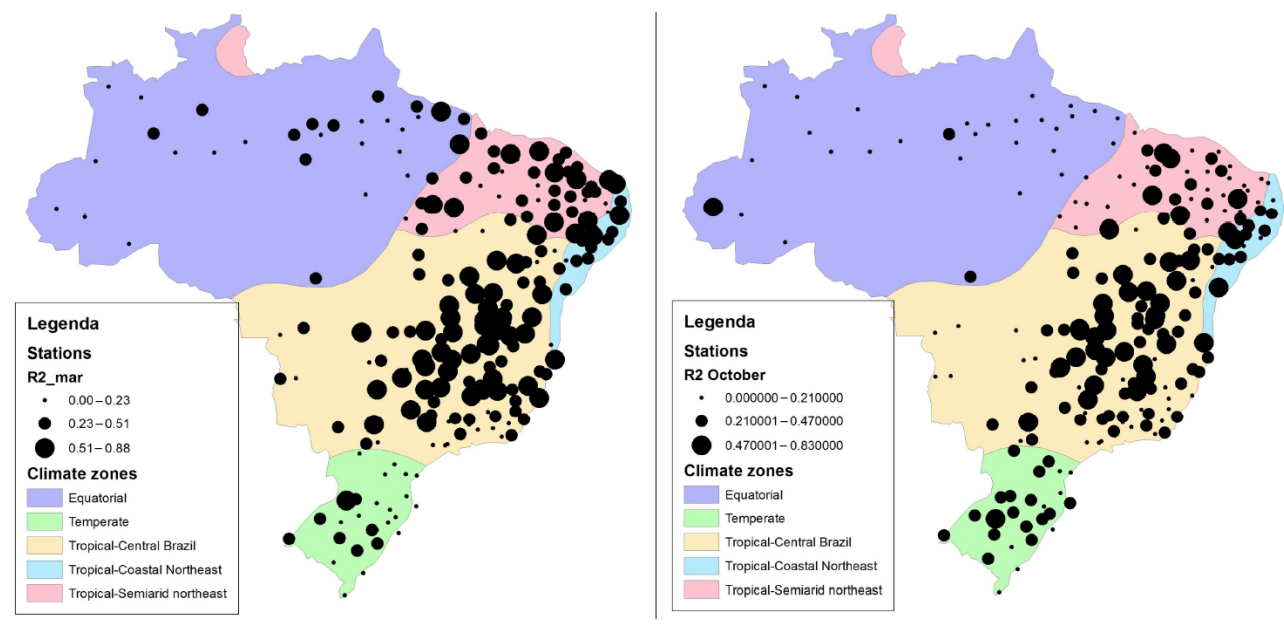

Figure 2. Spatialized values of $R^{2}$ statistic for transition months: March (left) and October (right).

Finally, Table 8 shows the average of $R^{2}$ statistics for each zone and month.

As mentioned, the winter months in the Southern Hemisphere (June to August) showed the lowest average regression performance, because of the low variability of rainfall during these months in most regions of Brazil.

\section{Conclusions}

This work is a first attempt to further explore the meteorological time series recently made available by INMET, based on a hydrological point of view. In the following sections, the main specific conclusions are presented.

\subsection{Statistical correspondence between explaining meteorological variables}

The statistical analysis performed over the meteorological variables explaining the evapotranspiration process sup- ported the comprehension of the mechanisms behind the inverse relationship between rainfall and ETP. Incoming radiation, which is the main driver of ETP, is clearly higher during dry days than in rainy days. Relative humidity, which also strongly influences ETP, is clearly higher during rainy days. Both results are intuitive and are supported by the statistical analysis.

Mean air temperature, which is also positively correlated with ETP, is generally higher during dry days, in comparison to rainy days. The results also showed that minimum temperatures very often were higher during dry days, mostly in the tropics. This result shows that one very popular premise of hydrometeorology is not necessarily true for tropical regions, namely the belief that minimum temperatures should be lower during clear weather due to less heat retention in the lower atmosphere.

For wind velocity and atmospheric pressure, results were less clear. In most cases, null hypothesis of equal means 
could not be rejected, thus not allowing further conclusions. One singular fact that was observed is a tendency for stronger wind during dry days in coastal places, while in hilly locations wind was stronger in rainy days.

Thus, the inverse relationship between rainfall and ETP is explained mostly by the higher incoming radiation (or sunshine duration) and lower relative humidity in dry days, and by a lesser part, by higher mean air temperature in these conditions.

\subsection{Regressions between rainfall and ETP}

In general, the results of the regressions confirmed the inverse relationship between rainfall and ETP, since regression slopes were negative in most cases.

Although the numerical values of the coefficient of determination $R^{2}$ were low, it must be remembered that these regressions are an alternative to the use of ETP averages, i.e. monotonical ETP values, in order to take at least a share of temporal variability into account. Thus, while the obtained regressions explain around $50 \%$ of ETP's variability, the usage of long-term averages would explain $0 \%$.

The obtained relations are particularly useful in tropical regions, during months of transition between rain and dry seasons, which can be either very wet or very dry, thus showing varying ETP.

These indirect estimates of ETP can be useful, for example, for extending ETP series into the past, in regions or periods where rainfall data is more abundant than meteorological data. As mentioned, the Brazilian hydropower network is planned and operated to supply energy demand, at a low risk, even in the case of a repetition of a critical hydrological period occurred in the 1950 s, when hydrometeorological data was scarce nationwide (and even more so in low-populated areas like the amazon, where hydropower was expanded in recent years). More accurate ETP estimates would be helpful both for a better assessment of inflows during that period (given the need of proper rainfall-runoff modelling) and for a better estimation of evaporation losses.
Other potential areas of usage are reservoir design and reservoir yield estimation, where long-term ETP averages are still widely used, leading to an overestimation of reservoir yields. Irrigation management would also be an area for application, through a better estimation of crop water needs, based only on rainfall measurements and the regressions obtained. Finally, assessment of future hydrological conditions would also benefit, since ETP conditions could be easily estimated based only on rainfall predictions.

\section{Data availability}

Meteorological data used in this paper belongs to the Brazilian Meteorological Institute and is available (after registration) at http://www.inmet.gov.br/projetos/rede/pesquisa/ inicio.php.

Acknowledgements. The authors thank the Brazilian National Institute of Meteorology (INMET) for making the hydrometerological time series available for academic purposes.

\section{References}

Allen, R., Pereira, L., Raes, D., and Smith, M.: Guidelines for computing crop water requirements. Rome: FAO, 310 pp. (FAOIrrigation and Drainage Paper, 56), 1998.

Brazilian Meteorological Institute: BDMEP: Meteorological Database for Research Purposes, available at: http://www.inmet.gov.br/projetos/rede/pesquisa/inicio.php, last access: December 2013.

Collischonn, W., Allasia, D., Silva, B., and Tucci, C. The MGBIPH model for large-scale rainfall-runoff modelling, Hydrol. Sci. J., 52, 878-895, 2007.

Instituto Brasileiro de Geografia e Estatística: Map of Brazilian Climate Zones, available at: ftp://geoftp.ibge.gov.br/informacoes_ ambientais/climatologia/mapas/brasil/clima.pdf, last access: December 2013.

Mudiare, O. J.: Influence of light rainfall and cloud cover on evapotranspiration demands, $\mathrm{PhD}$ Thesis, University of Saskatchewan, 1985.

Shuttleworth, W. J.: Terrestrial Hydrometeorology, WileyBlackwell, 448 pp., 2012.

Smith, M.: Report on the expert consultation on revision of FAO methodologies for crop water requirements, Technical Report, FAO/AGL, Rome, 1991.

Tucci, C.: Modelos Hidrológicos, UFRGS/ABRH, 678 pp., 1998 (in Portuguese). 